0204 MESOAMERICAN NEPHROPATHY IN COSTA RICA: GEOGRAPHICAL DISTRIBUTION AND TIME TRENDS OF CHRONIC KIDNEY DISEASE MORTALITY BETWEEN 1970 AND 2012

${ }^{1}$ Catharina Wesseling, ${ }^{2}$ Berna van Wendel de Joode, ${ }^{2}$ Jennifer Crowe, ${ }^{3}$ Ralf Rittner, ${ }^{3}$ Kristina Jakobsson. 'Institute of Environmental Medicine, Karolinska Institutet, Stockholm, Sweden; ${ }^{2}$ Central American Program on Work, Health and Environment (SALTRA), Universidad Nacional, Heredia, Costa Rica; ${ }^{3}$ Department of Occupational and Environmental Medicine, Lund University, Lund, Sweden

10.1136/oemed-2014-102362.83

Objectives To characterise geographical distribution and time trends of chronic kidney disease (CKD) mortality in the context of the epidemic of Mesoamerican nephropathy $(\mathrm{MeN})$, likely related to occupational heat stress and other, unknown, factors. Method Vital statistics (1970-2012) provided deaths from CKD and unspecified renal failure. Data of four censuses were extrapolated to derive person-years by sex and 10-year age groups for the seven provinces and 81 counties. SMRs were compared for three time periods between provinces and between counties, with national rates as reference. To assess time trends, age-specific and age-standardised mortality rates were computed for 5 -year periods.

Results During 1970-2012, 3843 men and 2452 women died from CKD. In the Guanacaste province, the SMR for 19972012 was four-fold in men and two-fold in women. In Guanacaste, CKD mortality increased from the mid-1970s in men, and mid-1980s in women. Age-standardised rates per 100.000 in men aged $\geq 30$ increased from 5.8 in the early seventies to 75.0 in 2007-2012, compared to 5.9 to 16.2 in the rest of Costa Rica. For women, rates increased from 4.5 to 20.7 in Guanacaste versus 4.2 to 9.7 in the rest of the country. Within Guanacaste, there was marked spatial variation in mortality between counties, with patterns being consistent between time periods but different for men and women.

Conclusions Guanacaste is a heterogeneous CKD "hot spot," affecting mostly men, but to lesser extent also women. CKD seemed high already four decades ago in the province. These findings are pertinent for etiologic research.

\section{LUNG CANCER RISK AMONG BRICKLAYERS IN A POOLED ANALYSIS OF CASE-CONTROL STUDIES}

${ }^{1}$ Dario Consonni, ${ }^{2}$ Sara De Matteis, ${ }^{3}$ Ann Olsson, ${ }^{4}$ Beate Pesch, ${ }^{5}$ Hans Kromhout, ${ }^{3}$ Kurt Straif, ${ }^{4}$ Thomas Brüning, ${ }^{6}$ SYNERGY Working Group. ${ }^{1}$ Epidemiology Unit, Fondazione IRCCS Ca' Granda - Ospedale Maggiore Policlinico, Milan, Italy; ${ }^{2}$ National Heart and Lung Institute, Occupational and Environmental Medicine, Imperial College London, London, UK; ${ }^{3}$ International Agency for Research on Cancer, Lyon, France; ${ }^{4}$ Institute for Prevention and Occupational Medicine of the German Social Accident Insurance Institute of the Ruhr-Universität Bochum (IPA), Bochum, Germany; ${ }^{5}$ Institute for Risk Assessment Sciences, Utrecht, The Netherlands; ${ }^{6}$ SYNERGY Working Group, France

\subsection{6/oemed-2014-102362.84}

Objectives Bricklayers may be exposed to several lung carcinogens, including crystalline silica and asbestos. Previous studies reported an excess of lung cancer among these workers. We examined lung cancer risk among bricklayers within SYNERGY, a large international pooled analysis of case-control studies on lung cancer and the joint effects of occupational carcinogens (http://SYNERGY.iarc.fr).

Method The pooled dataset included 15608 cases and 18531 controls from 22 centres in Europe, Canada, Hong Kong, and New Zealand. For men ever employed as bricklayers we estimated odds ratios (ORs) and 95\% confidence intervals (CIs) adjusted for study centre, age, lifetime cigarette smoking history, and employment in occupations with exposures to known or suspected lung carcinogens.

Results We found 1322 cases and 1004 controls who had ever worked as bricklayers (OR: 1.35; 95\% CI: 1.22-1.49). There was a clear positive trend with length of employment $(\mathrm{P}<$ 0.0001 ). The relative risk was higher for squamous (OR: 1.44, 95\% CI: 1.28-1.63, 578 cases) and small cell carcinomas (OR: 1.60, 95\% CI: 1.36-1.87, 248 cases), than for adenocarcinoma (OR: 1.14, 95\% CI: 0.98-1.32, 289 cases) (P-value for homogeneity: 0.0007). ORs were still elevated after additional adjustment for education and in analyses using blue collar workers as referents.

Conclusions This study provided additional evidence of increased lung cancer risk in bricklayers. Although non-causal explanations cannot be completely ruled out, the association is plausible in view of the potential for exposure to several carcinogens, notably crystalline silica and to a lesser extent asbestos.

\section{PRE-EXISTING HEALTH CONDITIONS AND JOB CHOICE AMONG YOUNG ADULTS IN THE ALSPAC COHORT}

${ }^{1}$ Kin Bong Hubert Lam, ${ }^{2}$ John Henderson, 'Jon G Ayres. 'University of Birmingham, Birmingham, UK: ${ }^{2}$ University of Bristol, Bristol, UK

\subsection{6/oemed-2014-102362.85}

Objectives "Traditional" occupational health research has focused on hazard identification in the work environment and the effects on health. Young adults represent $13 \%$ of the UK working population, yet little is known about whether pre-existing conditions are associated with their job choice.

Method The study was based on data from the Avon Longitudinal Study of Parents and Children (ALSPAC). At 16 and 18 years, participants were asked to report their current employment. Information on pre-existing asthma was obtained from previous questionnaires. Multivariate analysis was applied to determine the relationship between previous illness and current occupations.

Results A total of 5087 and 3347 participants responded to the 16 and 18 years questionnaires, respectively. At 16, 4.3\% left full time education for employment and $26.0 \%$ at 18 . Perceived overall health was slightly better among those still in education at 16 years, although such difference was no longer observed at 18 . Those with a previous diagnosis of asthma were less likely to be employed in jobs associated with high risk of adult onset asthma $(\mathrm{OR}=0.78 ; 95 \%$ CI $0.59-1.02)$. On the other hand, those who reported to have asthma at 16 but not at 18 were more likely to be engaged in high risk jobs $(\mathrm{OR}=2.20 ; 1.35-3.58)$.

Conclusions Results of this prospective study suggest a possible "healthy hire effect" among young people with asthma, although such avoidance might be modulated by the time of diagnosis.

\section{SOLAR ULTRAVIOLET RADIATION (UVR) EXPOSURE LEVELS AND SUN PROTECTION BEHAVIOURS IN OUTDOOR WORKERS IN BRITISH COLUMBIA, CANADA}

${ }^{1}$ Cheryl Peters, ${ }^{1}$ Sunil Kalia, ${ }^{2}$ Paul Demers, ${ }^{3}$ Anne-Marie Nicol, ${ }^{1}$ Mieke Koehoorn. ${ }^{1}$ University of British Columbia, Vancouver, British Columbia, Canada; ${ }^{2}$ Occupational Cancer Research Centre, Toronto, Ontario, Canada; ${ }^{3}$ Simon Fraser University, Burnaby, British Columbia, Canada

10.1136/oemed-2014-102362.86 